\title{
FY10 Parameters for the Injection, Acceleration, and Extraction of Gold Ions in Booster, AGS, and RHIC
}

\author{
C.J. Gardner \\ Brookhaven National Laboratory, Upton, NY 11973, USA
}

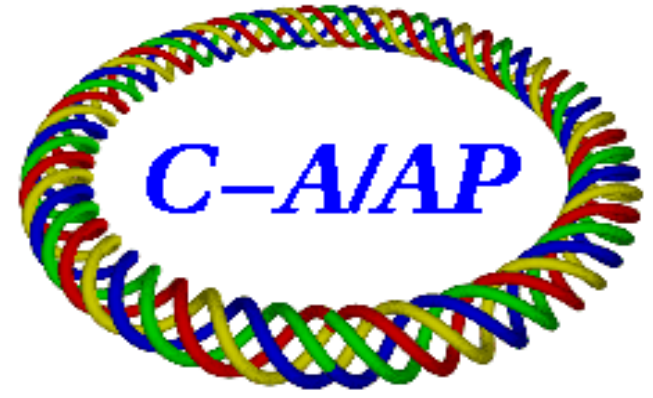

\section{Collider-Accelerator Department Brookhaven National Laboratory Upton, NY 11973}

Notice: This document has been authorized by employees of Brookhaven Science Associates, LLC under Contract No. DE-AC02-98CH10886 with the U.S. Department of Energy. The United States Government retains a nonexclusive, paid-up, irrevocable, world-wide license to publish or reproduce the published form of this document, or allow others to do so, for United States Government purposes. 


\title{
FY10 Parameters for the Injection, Acceleration, and Extraction of Gold Ions in Booster, AGS, and RHIC
}

\author{
C.J. Gardner
}

April 15, 2010

\section{Basic Parameters}

A Gold ion with charge $e Q$ has $N=197$ Nucleons, $Z=79$ Protons, and $(Z-Q)$ electrons. (Here $Q$ is an integer and $e$ is the charge of a single proton.) The mass is

$$
m=a u-Q m_{e}+E_{b} / c^{2}
$$

where $a=196.966552$ is the relative atomic mass $[1,2]$ of the neutral Gold atom, $u=931.494013 \mathrm{MeV} / c^{2}$ is the unified atomic mass unit [3], and $m_{e} c^{2}=.510998902 \mathrm{MeV}$ is the electron mass [3]. $E_{b}$ is the binding energy of the $Q$ electrons removed from the neutral Gold atom. This amounts to $0.332 \mathrm{MeV}$ for the helium-like gold ion $(Q=77)$ and $0.517 \mathrm{MeV}$ for the fully stripped ion. For the $\mathrm{Au}^{31+}$ ion we have $E_{b}=13.5 \mathrm{keV}$. These numbers are given in Ref. [4].

In a circular accelerator the ion moves along an orbit of circumference $2 \pi R$ with revolution frequency $f$. The radius of the orbit is $R$. The velocity of the ion is then

$$
v=2 \pi R f .
$$

This gives momentum, energy, and kinetic energy

$$
p=m c \beta \gamma, \quad E=m c^{2} \gamma=\sqrt{p^{2} c^{2}+m^{2} c^{4}}, \quad W=E-m c^{2}
$$

where

$$
\beta=v / c, \quad \gamma=1 / \sqrt{1-\beta^{2}} .
$$


The magnetic rigidity of the ion in units of $\mathrm{Tm}$ is

$$
B \rho=k p / Q
$$

where $k=10^{9} / 299792458$ and $p$ is the momentum in units of $\mathrm{GeV} / \mathrm{c}$. The angular frequency is

$$
\omega=2 \pi f .
$$

We also define the phase-slip factor

$$
\eta=\frac{1}{\gamma_{t}^{2}}-\frac{1}{\gamma^{2}}
$$

where $\gamma_{t}$ is the transition gamma.

\section{RF Parameters}

The stationary bucket area is

$$
A_{S}=8 \frac{R_{s}}{h c}\left\{\frac{2 e Q V_{g} E_{s}}{\pi h\left|\eta_{s}\right|}\right\}^{1 / 2}
$$

where $h$ is the RF harmonic number, $V_{g}$ is the total RF gap voltage per turn, and the subscript " $s$ " denotes parameter values for the synchronous particle.

The half-height of a bucket is

$$
\Delta E=\left(\frac{h \omega_{s}}{8 \sqrt{2}}\right) A_{S}\left|\left(\pi-2 \phi_{s}\right) \sin \phi_{s}-2 \cos \phi_{s}\right|^{1 / 2}
$$

where $\phi_{s}$ is the synchronous phase.

The synchronous phase is given by

$$
V_{g} \sin \phi_{s}=2 \pi R_{s} \rho_{s} \dot{B} / c
$$

where $\rho_{s}$ is the radius of curvature, $B$ is the magnetic field and $\dot{B}=d B / d t$. Employing Gaussian units $\left(R_{s}\right.$ and $\rho_{s}$ in $\mathrm{cm}$, $c=2.99792458 \times 10^{10} \mathrm{~cm} / \mathrm{s}$, and $\dot{B}$ in $\left.\mathrm{G} / \mathrm{s}\right)$ gives $V_{g} \sin \phi_{s}$ in Statvolts. Multiplying by 299.792458 then gives $V_{g} \sin \phi_{s}$ in Volts.

The width of a bucket is

$$
\Delta t=\frac{\left|\pi-\phi_{s}-\phi_{e}\right|}{h \omega_{s}}
$$


where the phase $\phi_{e}$ satisfies

$$
\cos \left(\pi-\phi_{s}\right)-\cos \phi_{e}=-\left(\pi-\phi_{s}-\phi_{e}\right) \sin \phi_{s} .
$$

The area of a bucket is

$$
A_{\mathrm{bk}}=\alpha\left(\phi_{s}\right) A_{S}
$$

where

$$
\alpha\left(\phi_{s}\right)=\frac{\sqrt{2}}{8} \int_{\phi_{L}}^{\phi_{R}}\left|\left(\pi-\phi_{s}-\phi\right) \sin \phi_{s}-\cos \phi_{s}-\cos \phi\right|^{1 / 2} d \phi .
$$

Below transition we have $\phi_{e}<\pi-\phi_{s}$ and the limits of integration are $\phi_{L}=\phi_{e}$ and $\phi_{R}=\pi-\phi_{s}$. Above transition we have $\pi-\phi_{s}<\phi_{e}$ and the limits of integration are $\phi_{L}=\pi-\phi_{s}$ and $\phi_{R}=\phi_{e}$. The integral $\alpha\left(\phi_{s}\right)$ must be evaluated numerically. An approximate expression is [5]

$$
\alpha\left(\phi_{s}\right) \approx \frac{1-\sin \phi_{s}}{1+\sin \phi_{s}}
$$

The synchrotron frequency for small-amplitude oscillations about $\phi_{s}$ is

$$
F_{s}=\frac{c}{2 \pi R_{s}}\left\{\frac{-h \eta_{s} e Q V_{g} \cos \phi_{s}}{2 \pi E_{s}}\right\}^{1 / 2}
$$

and the corresponding synchrotron tune is $Q_{s}=2 \pi F_{s} / \omega_{s}$. Note that measurement of $F_{s}$ gives a value for $V_{g} \cos \phi_{s}$, while measurement of $d B / d t$ gives a value for $V_{g} \sin \phi_{s}$. These can be used to obtain $V_{g}$ and $\phi_{s}$.

Let $\phi_{l}$ and $\phi_{r}$ be the phases at the left and right boundries of a bunch matched to a bucket. We have

$$
\phi_{l}<\phi_{s}<\phi_{r}
$$

and the width of the bunch is

$$
\Delta t=\frac{\Delta \phi}{h \omega_{s}}, \quad \Delta \phi=\phi_{r}-\phi_{l} .
$$

In terms of $\Delta \phi$ and $\phi_{s}$ we have

$$
\phi_{r}=\frac{\Delta \phi}{2}+\arcsin \left\{\frac{\Delta \phi \sin \phi_{s}}{2 \sin (\Delta \phi / 2)}\right\}
$$

and

$$
\phi_{l}=-\frac{\Delta \phi}{2}+\arcsin \left\{\frac{\Delta \phi \sin \phi_{s}}{2 \sin (\Delta \phi / 2)}\right\} .
$$


Note that if $\Delta \phi$ is small we have

$$
\sin (\Delta \phi / 2) \approx \frac{\Delta \phi}{2}, \quad \frac{\Delta \phi \sin \phi_{s}}{2 \sin (\Delta \phi / 2)} \approx \sin \phi_{s}
$$

and

$$
\phi_{l} \approx \phi_{s}-\frac{\Delta \phi}{2}, \quad \phi_{r} \approx \phi_{s}+\frac{\Delta \phi}{2} .
$$

The half-height of the bunch is

$$
\Delta E=\left(\frac{h \omega_{s}}{8 \sqrt{2}}\right) A_{S}\left|\cos \phi_{r}-\cos \phi_{s}+\left(\phi_{r}-\phi_{s}\right) \sin \phi_{s}\right|^{1 / 2} .
$$

The area of the bunch is

$$
A_{\mathrm{b}}=F\left(\phi_{s}, \Delta \phi\right) A_{S}
$$

where

$$
F\left(\phi_{s}, \Delta \phi\right)=\frac{\sqrt{2}}{8} \int_{\phi_{l}}^{\phi_{r}}\left|\cos \phi_{l}-\cos \phi+\left(\phi_{l}-\phi\right) \sin \phi_{s}\right|^{1 / 2} d \phi .
$$

The integral $F\left(\phi_{s}, \Delta \phi\right)$ must be evaluated numerically. If $\Delta \phi$ is small we have

$$
F\left(\phi_{s}, \Delta \phi\right) \approx \frac{\pi}{64}(\Delta \phi)^{2}\left|\cos \phi_{s}\right|^{1 / 2} .
$$

\section{$3 \quad$ Ring Parameters}

\begin{tabular}{|c|c|c|c|c|}
\hline Parameter & Booster & AGS & RHIC & Unit \\
\hline$C_{I}$ & $C_{b}$ & $C_{a}$ & $C_{r}+\delta C$ & $\mathrm{~m}$ \\
\hline$C_{E}$ & $C_{a} / 4$ & $4\left(C_{r}+\delta C\right) / 19$ & $C_{r}+\delta C$ & $\mathrm{~m}$ \\
\hline$\rho$ & 13.8656 & 85.378351 & 242.7806 & $\mathrm{~m}$ \\
\hline$\gamma_{\mathrm{tr}}$ & 4.806 & 8.5 & 22.89 & \\
\hline
\end{tabular}

Here $C_{I}$ and $C_{E}$ are the circumferences of the closed orbits in the machines at injection and extraction (or store) respectively. $C_{b}, C_{a}$, and $C_{r}$ are the circumferences of the "design" orbits in Booster, AGS, and RHIC respectively. These are

$$
C_{b}=201.780, \quad C_{a}=2 \pi(128.4526), \quad C_{r}=3833.845181
$$


meters. $\delta C$ is the shift (if any) of the RHIC orbit circumference from the design value $C_{r}$. Note that $4\left(C_{r} / 19\right)=2 \pi(128.4580) \mathrm{m}$ which gives an AGS radius at extraction approximately $5 \mathrm{~mm}$ larger than the "design" AGS radius $(128.4526 \mathrm{~m})$ reported by Bleser $[6,7]$. The radius of curvature $\rho$ in the Booster and AGS main dipoles is given in Refs. $[6,7,8]$. The RHIC ring parameters are taken from Ref. [9] and from MAD runs by Steve Tepikian.

For Run 10 the crossing angle at Store in the four non-experimental Intersection Regions (IR10, IR12, IR2 and IR4) is 2 milliradians. This gives an orbit circumference less than $C_{r}$. The shift $\delta C$ has been calculated by Steve Tepikian and is given below.

\section{Assumptions}

The parameters values listed in Sections 5-11 are calculated assuming that:

1. The magnetic rigidity of the $\mathrm{Au}^{31+}$ ion and deuteron at Booster injection is $B \rho=0.8813444 \mathrm{Tm}$.

2. The magnetic rigidity of the $\mathrm{Au}^{31+}$ ion at Booster extraction is $B \rho=9.4307359 \mathrm{Tm}$.

3. The magnetic rigidity of the $\mathrm{Au}^{77+}$ ion at AGS injection is $B \rho=3.7474455 \mathrm{Tm}$.

4. The magnetic rigidity of the $\mathrm{Au}^{79+}$ ion at RHIC injection is the same as that of a proton with $\gamma_{p}$ such that $G \gamma_{p}=46.5$. Here $G+1=2.792847337(29)$ and the proton mass is $m_{p}=0.938271998(38) \mathrm{GeV} / c^{2}$ as reported in Ref. [10]. Thus

$\gamma_{p}=25.93639684$ and the proton momentum and energy are

$P_{p}=m_{p} c \sqrt{\gamma_{p}^{2}-1}=24.3173002 \mathrm{GeV} / \mathrm{c}$ and

$E_{p}=m_{p} c^{2} \gamma_{p}=24.3353949 \mathrm{GeV}$. The rigidity is then $B \rho=k P_{p}=81.1137824 \mathrm{Tm}$.

5. The circumference at RHIC injection is $C_{r}$.

6. The circumference at Store is $1.400 \mathrm{~mm}$ less than $C_{r}$.

7. The energy of the $\mathrm{Au}^{79+}$ ion at RHIC Store is $100 \mathrm{GeV}$ per nucleon. 
Please note that more digits are given for some parameters in Sections 5-11 than would be warranted by the precision with which the parameters could be measured; this is done for computational convenience. The notation " $N$ " in the Units column of the tables means "per nucleon".

\section{Gold Parameters in Booster}

\begin{tabular}{|c|c|c|c|}
\hline Parameter & Injection & Extraction & Unit \\
\hline$Q$ & 31 & 31 & \\
\hline$m$ & 183.457336 & 183.457336 & $\mathrm{GeV} / c^{2}$ \\
\hline$W$ & $182.75731 / 197$ & 100.81631 & $\mathrm{MeV} / N$ \\
\hline$c p$ & 41.577830 & 444.89933 & $\mathrm{MeV} / N$ \\
\hline$E$ & 0.93218322 & 1.0320718 & $\mathrm{GeV} / N$ \\
\hline$B \rho$ & 0.8813444 & 9.4307359 & $\mathrm{Tm}$ \\
\hline$\beta$ & 0.044602637 & 0.43107400 & \\
\hline$\gamma-1$ & $0.99618423 / 1000$ & 0.10825848 & \\
\hline$\eta$ & -0.955 & -0.771 & \\
\hline$\epsilon_{H}(95 \%)$ & $8.3 \pi$ & $8.3 \pi$ & $\mathrm{mm} \mathrm{mrad}$ \\
\hline$\epsilon_{V}(95 \%)$ & $3.9 \pi$ & $3.9 \pi$ & $\mathrm{mm} \mathrm{mrad}$ \\
\hline$h$ & 6 & 6 & \\
\hline$h f$ & 0.39760732 & 3.8429170 & $\mathrm{MHz}$ \\
\hline$R$ & $201.780 /(2 \pi)$ & $128.4526 / 4$ & $\mathrm{~m}$ \\
\hline
\end{tabular}

Here $\epsilon_{H}$ and $\epsilon_{V}$ are the normalized horizontal and vertical transverse emittances. These follow from the assumption that during multi-turn injection the horizontal and vertical acceptances in Booster are completely filled. The horizontal and vertical acceptances are $185 \pi$ and $87 \pi \mathrm{mm} \mathrm{mrad}$ (un-normalized) respectively.

\begin{tabular}{|c|c|c|c|}
\hline Parameter & Injection & Extraction & Unit \\
\hline$V_{g}$ & 0.5 & 30 & $\mathrm{kV}$ \\
$A_{S}$ & 2.540 & 23.04 & $\mathrm{eV} \mathrm{s}$ \\
$d B / d t$ & 0 & 80.0 & $\mathrm{G} / \mathrm{ms}$ \\
\hline$\phi_{s}$ & 0 & 48.25 & degrees \\
$F_{s}$ & 0.412 & 2.225 & $\mathrm{kHz}$ \\
$A_{\mathrm{bk}}$ & 2.540 & 3.111 & $\mathrm{eV} \mathrm{s}$ \\
\hline$A_{b}$ & 0.7263 & 1.499 & $\mathrm{eV} \mathrm{s}$ \\
$\Delta t$ & 1008 & 55.0 & $\mathrm{~ns}$ \\
$\Delta E$ & 0.467 & 17.7 & $\mathrm{MeV}$ \\
\hline
\end{tabular}




\begin{tabular}{|c|c|c|c|}
\hline Parameter & Injection & Extraction & Unit \\
\hline No. of Bunches & 6 & 6 & \\
\hline Bunch Spacing & 2515.044 & 260.2190 & $\mathrm{~ns}$ \\
\hline Ions/Bunch & $3.47 / 6$ & $2.98 / 6$ & $10^{9}$ \\
\hline Bunch Area & $0.0221 / 6$ & $0.0457 / 6$ & $\mathrm{eV} \mathrm{s} / N$ \\
\hline
\end{tabular}

At Booster injection, the voltage $V_{I}$ required for particles with momentum $p$ and charge $Q$ to follow the nominal trajectory through the inflector is given by

$$
e V_{I}=\frac{G}{R_{I}}\left(\frac{c^{2} p^{2}}{Q E}\right)
$$

Here $G=0.017 \mathrm{~m}$ is the gap between the cathode and septum of the inflector and $R_{I}=8.74123 \mathrm{~m}$ is the radius of curvature along the nominal trajectory. Using the tabulated values of $c p$ and $E$ at Booster injection, we obtain $V_{I}=22.919 \mathrm{kV}$.

The fractional momentum spread $\Delta p / p$ was measured by chopping a short notch out of the unbunched beam in the Tandem-to-Booster (TTB) transfer line, and observing the turn-by-turn spreading of the notch in Booster at injection. This gives $\Delta p / p= \pm 3.9 \times 10^{-4}$. Observation of the notch also gives $15.1 \mu \mathrm{s}$ for the revolution period at injection. The longitudinal emittance of the unbunched beam after accumulation in Booster is then $0.022 \mathrm{eV}$-s per nucleon.

Capture of injected beam in Booster occurs on a $6 \mathrm{~ms}$ porch at constant field. During this time the gap voltage is increased from 0 to $0.5 \mathrm{kV}$, capturing the beam into $h=6$ stationary RF buckets. The tabulated bunch area at extraction was determined from measurements of the bunch width (55 ns) at extraction with $d B / d t=80 \mathrm{G} / \mathrm{ms}$ and $V_{g}=30 \mathrm{kV}$. The tabulated intensities (ions per bunch) were obtained during the FY 2007 RHIC run [11] with $5.37 \times 10^{9}$ ions per pulse at the end of the TTB line.

The six bunches are extracted from Booster in a single turn by means of a fast kicker and ejector septum magnet. Measurements of the beam width just downstream of the ejector give $95 \%$ horizontal and vertical emittances $4.2 \pi$ and $2.8 \pi$ ( $\mathrm{mm}$ milliradians) respectively. After extraction, the ions pass through a stripper in the Booster to AGS (BTA) transport line where approximately $60 \%$ emerge in charge state +77 . The stripper consists of a $6.35 \mathrm{mg} / \mathrm{cm}^{2}$ aluminum foil followed by a $8.48 \mathrm{mg} / \mathrm{cm}^{2}$ "glassy" carbon foil mounted just downstream [12]. The thicknesses have been optimized to produce the highest yield of $\mathrm{Au}^{77+}$. The high uniformity of the glassy 
carbon, compared to that of the standard carbon stripper $\left(23.1 \mathrm{mg} / \mathrm{cm}^{2}\right.$ graphite) used in the past, gives a significant reduction in the increase of longitudinal emittance due variable energy loss as the ions traverse the foil. With the standard carbon foil, this increase was approximately a factor of four; with the glassy carbon, the increase is a factor of 1.8. The measured energy spread of the bunches in Booster at extraction is $\pm 18 \mathrm{MeV}$ while that of the bunches in AGS at injection is $\pm 32 \mathrm{MeV}$. The measured average energy loss in the foils is $2.5 \mathrm{MeV}$ per nucleon. This is significantly less the the $4 \mathrm{MeV}$ per nucleon observed with the standard carbon stripper.

The $\mathrm{Au}^{77+}$ ions are injected into the AGS by means of a septum magnet and a fast kicker. Four batches of six bunches are injected at constant magnetic field to give a total of 24 bunches on the AGS injection porch.

The bunches are injected into stationary buckets at harmonic 24. Because of the reduced energy spread of ions emerging from the BTA stripper used now, there is more than enough voltage available to match the buckets to the incoming bunches. (This was not possible with the standard carbon foil used in the past.) The required voltage is approximately $100 \mathrm{kV}$ per turn. Measurements of bunch width ( $55 \mathrm{~ns}$ ) in the matched buckets give a six-bunch longitudinal emittance of $0.082 \mathrm{eV}$-s per nucleon. This is a factor of 1.8 greater than the emittance measured at Booster extraction. In addition to emittance growth due to variable energy loss as ions traverse the foil there is a phase mismatch caused by the average energy loss. Since the ions emerge from the foil with a smaller average velocity, the distance between bunch centers is reduced. (The time between bunch centers is unchanged.) This means that the 6 bunches of each batch entering the AGS will occupy slightly less than one fourth of the ring. The effect of the mismatch is to cause some dilution of longitudinal emittance during the merging process discussed below.

In the past, shortly after all four batches from Booster were injected, the harmonic 24 voltage was slowly reduced, adiabatically debunching the beam. Once debunched the beam was adiabatically rebunched into 4 bunches (in order to reach the bunch intensity desired for RHIC) and then accelerated to top energy at harmonic 12. Experience with this setup has shown that there can be beam instability and subsequent fast loss due to the small momentum spread of the unbunched beam. To avoid this, a new setup in which the 24 bunches are merged into 4 was developed and implemented for the FY 2007 RHIC run [11]. The merge is done in two steps. First the 24 bunches are merged into 12 by bringing on harmonic 12 while reducing harmonic 24 . Then the 12 bunches are merged into 4 by 
bringing on harmonics 4 and 8 while reducing harmonic 12. This final merge is done with a single low-frequency cavity. The resulting 4 equally spaced bunches are then accelerated to top energy at harmonic 12 .

\section{$6 \quad$ Booster Injection Field}

The magnetic field at Booster injection is measured with a Hall probe and the Booster Gauss Clock. The Hall probe sits in the reference dipole and gives the value of the field at BT0. The Gauss Clock gives the increment in field between BT0 and the time of injection. The measured field at injection is defined to be the field at BT0 plus the field increment. For the injection of $\mathrm{Au}^{31+}$ ions, the measured field is 629.5 Gauss.

\section{Gold Parameters in AGS}

\begin{tabular}{|c|c|c|c|c|}
\hline Parameter & Injection & Transition & Extraction & Unit \\
\hline$Q$ & 77 & 77 & 77 & \\
\hline$m$ & 183.434149 & 183.434149 & 183.434149 & $\mathrm{GeV} / c^{2}$ \\
\hline$W$ & 0.098348279 & 6.9835336 & 8.8648684 & $\mathrm{GeV} / N$ \\
\hline$c p$ & 0.43911728 & 7.8597077 & 9.7516522 & $\mathrm{GeV} / N$ \\
\hline$E$ & 1.0294861 & 7.9146714 & 9.7960062 & $\mathrm{GeV} / N$ \\
\hline$B \rho$ & 3.7474455 & 67.075079 & 83.2210136 & $\mathrm{Tm}$ \\
\hline$\beta$ & 0.42654028 & 0.99305547 & 0.99547223 & \\
\hline$\gamma$ & 1.1056216 & 8.5000 & 10.520469 & \\
\hline$\eta$ & -0.804 & 0.0 & 0.00481 & \\
\hline$\epsilon_{H}(95 \%)$ & $\leq 10 \pi$ & $\leq 10 \pi$ & $\leq 10 \pi$ & $\mathrm{mm} \mathrm{mrad}$ \\
\hline$\epsilon_{V}(95 \%)$ & $\leq 10 \pi$ & $\leq 10 \pi$ & $\leq 10 \pi$ & $\mathrm{mm} \mathrm{mrad}$ \\
\hline$h$ & 24 & 12 & 12 & \\
\hline$h f$ & 3.8025000 & 4.42642072 & 4.43700724 & $\mathrm{MHz}$ \\
\hline$R$ & 128.4526 & 128.4526 & 128.45798 & $\mathrm{~m}$ \\
\hline
\end{tabular}




\begin{tabular}{|c|c|c|c|}
\hline Parameter & Injection & Extraction & Unit \\
\hline$V_{g}$ & 101.4 & 179 & $\mathrm{kV}$ \\
$A_{S}$ & 32.64 & 4895 & $\mathrm{eV} \mathrm{s}$ \\
$d B / d t$ & 0 & 0 & $\mathrm{G} / \mathrm{ms}$ \\
\hline$\phi_{s}$ & 0 & 180 & degrees \\
$F_{s}$ & 4.039 & 0.0951 & $\mathrm{kHz}$ \\
$A_{\mathrm{bk}}$ & 32.64 & 4895 & $\mathrm{eV} \mathrm{s}$ \\
\hline$A_{b}$ & 2.705 & $6 \times 7.653$ & $\mathrm{eV} \mathrm{s}$ \\
$\Delta t$ & 55.0 & 15.7 & $\mathrm{~ns}$ \\
$\Delta E$ & 31.5 & 1863 & $\mathrm{MeV}$ \\
\hline
\end{tabular}

\begin{tabular}{|c|c|c|c|}
\hline Parameter & Injection & Extraction & Unit \\
\hline No. of Bunches & 24 & 4 & \\
\hline Bunch Spacing & 262.985 & 676.131 & $\mathrm{~ns}$ \\
\hline Ions/Bunch & $1.70 / 6$ & 1.68 & $10^{9}$ \\
\hline Bunch Area & $0.0824 / 6$ & 0.233 & $\mathrm{eV} \mathrm{s} / N$ \\
\hline
\end{tabular}

Measurements of bunch width (16 ns) and gap volts per turn $(180 \mathrm{kV})$ just before extraction give a single-bunch longitudinal emittance of $0.23 \mathrm{eV}$-s per nucleon.

\section{Gold Parameters in RHIC}

\begin{tabular}{|c|c|c|c|c|}
\hline Parameter & Injection & Transition & Store & Unit \\
\hline$Q$ & 79 & 79 & 79 & \\
\hline$m$ & 183.433312 & 183.433312 & 183.433312 & $\mathrm{GeV} / c^{2}$ \\
\hline$W$ & 8.8648280 & 20.382514 & 99.068866 & $\mathrm{GeV} / N$ \\
\hline$c p$ & 9.7516077 & 21.293298 & 99.995665 & $\mathrm{GeV} / N$ \\
\hline$E$ & 9.7959615 & 21.313647 & 100.000000 & $\mathrm{GeV} / N$ \\
\hline$B \rho$ & 81.1137824 & 177.117457 & 831.763013 & $\mathrm{Tm}$ \\
\hline$\beta$ & 0.99547223 & 0.99904526 & 0.99995665 & \\
\hline$\gamma$ & 10.520469 & 22.8900 & 107.395978 & \\
\hline$\eta$ & -0.00713 & 0.0 & 0.00182 & \\
\hline$\epsilon_{H}(95 \%)$ & $\leq 10 \pi$ & $\leq 10 \pi$ & $\leq 10 \pi$ & $\mathrm{mm} \mathrm{mrad}$ \\
\hline$\epsilon_{V}(95 \%)$ & $\leq 10 \pi$ & $\leq 10 \pi$ & $\leq 10 \pi$ & $\mathrm{mm} \mathrm{mrad}$ \\
\hline$h$ & 360 & 360 & 2520 & \\
\hline$h f$ & 28.0232036 & 28.1237867 & 197.0461725 & $\mathrm{MHz}$ \\
\hline$\delta C$ & 0.0 & 0.0 & -1.400 & $\mathrm{~mm}$ \\
\hline
\end{tabular}




\begin{tabular}{|c|c|c|c|}
\hline Parameter & Transition & Transition & Unit \\
\hline Ring & Yellow & Blue & \\
\hline$Q$ & 79 & 79 & \\
\hline$m$ & 183.433312 & 183.433312 & $\mathrm{GeV} / c^{2}$ \\
\hline$W$ & 22.998999 & 23.418009 & $\mathrm{GeV} / N$ \\
\hline$c p$ & 23.912010 & 24.331332 & $\mathrm{GeV} / N$ \\
\hline$E$ & 23.930133 & 24.349143 & $\mathrm{GeV} / N$ \\
\hline$B \rho$ & 198.89988 & 202.38780 & $\mathrm{Tm}$ \\
\hline$\beta$ & 0.99924270 & 0.99926855 & \\
\hline$\gamma$ & 25.70 & 26.15 & \\
\hline$\eta$ & 0.0 & 0.0 & \\
\hline$\epsilon_{H}(95 \%)$ & $\leq 10 \pi$ & $\leq 10 \pi$ & $\mathrm{mm} \mathrm{mrad}$ \\
\hline$\epsilon_{V}(95 \%)$ & $\leq 10 \pi$ & $\leq 10 \pi$ & $\mathrm{mm} \mathrm{mrad}$ \\
\hline$h$ & 360 & 360 & \\
\hline$h f$ & 28.1293448 & 28.1300725 & $\mathrm{MHz}$ \\
\hline$\delta C$ & 0.0 & 0.0 & $\mathrm{~mm}$ \\
\hline \multicolumn{4}{|c}{} \\
\hline
\end{tabular}

\begin{tabular}{|c|c|c|c|}
\hline Parameter & Injection & Store & Unit \\
\hline$V_{g}$ & 237.8 & 3000 & $\mathrm{kV}$ \\
$A_{S}$ & 135.7 & 164.4 & $\mathrm{eV} \mathrm{s}$ \\
$d B / d t$ & 0 & 0 & $\mathrm{G} / \mathrm{ms}$ \\
\hline$\phi_{s}$ & 0 & 180 & degrees \\
$F_{s}$ & 0.156 & 0.232 & $\mathrm{kHz}$ \\
$A_{\mathrm{bk}}$ & 135.7 & 164.4 & $\mathrm{eV} \mathrm{s}$ \\
\hline$A_{b}$ & 45.9 & 137.8 & $\mathrm{eV} \mathrm{s}$ \\
$\Delta t$ & 15.7 & 4.0 & $\mathrm{~ns}$ \\
$\Delta E$ & 1903 & 24048 & $\mathrm{MeV}$ \\
\hline
\end{tabular}

\begin{tabular}{|c|c|c|c|}
\hline Parameter & Injection & Store & Unit \\
\hline No. of Bunches & 60 & 60 & \\
\hline Bunch Spacing & 214.108 & 213.148 & $\mathrm{~ns}$ \\
\hline Ions/Bunch & 1.20 & 1.14 & $10^{9}$ \\
\hline Bunch Area & 0.233 & 0.70 & $\mathrm{eV} \mathrm{s} / N$ \\
\hline
\end{tabular}




\section{Gold Parameters in AGS}

\begin{tabular}{|c|c|c|c|c|}
\hline Parameter & Injection & Transition & Extraction & Unit \\
\hline$Q$ & 77 & 77 & 77 & \\
\hline$m$ & 183.434149 & 183.434149 & 183.434149 & $\mathrm{GeV} / c^{2}$ \\
\hline$W$ & 0.098348279 & 6.9835336 & 8.8648684 & $\mathrm{GeV} / N$ \\
\hline$c p$ & 0.43911728 & 7.8597077 & 9.7516522 & $\mathrm{GeV} / N$ \\
\hline$E$ & 1.0294861 & 7.9146714 & 9.7960062 & $\mathrm{GeV} / N$ \\
\hline$B \rho$ & 3.7474455 & 67.075079 & 83.2210136 & $\mathrm{Tm}$ \\
\hline$\beta$ & 0.42654028 & 0.99305547 & 0.99547223 & \\
\hline$\gamma$ & 1.1056216 & 8.5000 & 10.520469 & \\
\hline$\eta$ & -0.804 & 0.0 & 0.00481 & \\
\hline$\epsilon_{H}(95 \%)$ & $\leq 10 \pi$ & $\leq 10 \pi$ & $\leq 10 \pi$ & $\mathrm{mm} \mathrm{mrad}$ \\
\hline$\epsilon_{V}(95 \%)$ & $\leq 10 \pi$ & $\leq 10 \pi$ & $\leq 10 \pi$ & $\mathrm{mm} \mathrm{mrad}$ \\
\hline$h$ & 24 & 12 & 12 & \\
\hline$h f$ & 3.8025000 & 4.42642072 & 4.43700724 & $\mathrm{MHz}$ \\
\hline$R$ & 128.4526 & 128.4526 & 128.45798 & $\mathrm{~m}$ \\
\hline
\end{tabular}

\section{Medium-Energy Gold Parameters in RHIC}

\begin{tabular}{|c|c|c|c|c|}
\hline Parameter & Energy 1 & Energy 2 & Injection & Unit \\
\hline$Q$ & 79 & 79 & 79 & \\
\hline$m$ & 183.433312 & 183.433312 & 183.433312 & $\mathrm{GeV} / c^{2}$ \\
\hline$W$ & 30.268866 & 18.568866 & 8.8648280 & $\mathrm{GeV} / N$ \\
\hline$c p$ & 31.186103 & 19.477756 & 9.7516077 & $\mathrm{GeV} / N$ \\
\hline$E$ & 31.2 & 19.5 & 9.7959615 & $\mathrm{GeV} / N$ \\
\hline$B \rho$ & 259.40571 & 162.01580 & 81.1137824 & $\mathrm{Tm}$ \\
\hline$\beta$ & 0.99955457 & 0.99885930 & 0.99547223 & \\
\hline$\gamma$ & 33.507545 & 20.942216 & 10.520469 & \\
\hline$\eta$ & 0.001018 & -0.0003715 & -0.00713 & \\
\hline$h$ & 360 & 360 & 360 & \\
\hline$h f$ & 28.1381241 & 28.118552 & 28.0232036 & $\mathrm{MHz}$ \\
\hline$\delta C$ & 0 & 0 & 0 & $\mathrm{~mm}$ \\
\hline
\end{tabular}




\section{Low-Energy Gold Parameters at AGS Extraction}

\begin{tabular}{|c|c|c|c|c|c|}
\hline & Energy 1 & Energy 2 & Energy 3 & Energy 4 & Unit \\
\hline$Q$ & 77 & 77 & 77 & 77 & \\
\hline$m$ & 183.434149 & 183.434149 & 183.434149 & 183.434149 & $\mathrm{GeV} / c^{2}$ \\
\hline$W$ & 8.0689033 & 4.8188884 & 2.9188798 & 1.5688736 & $\mathrm{GeV} / N$ \\
\hline$c p$ & 8.9517441 & 5.6741329 & 3.7357218 & 2.3201378 & $\mathrm{GeV} / N$ \\
\hline$E$ & 9.0000411 & 5.7500262 & 3.8500176 & 2.5000114 & $\mathrm{GeV} / N$ \\
\hline$B \rho$ & 76.394564 & 48.423291 & 31.880809 & 19.800154 & $\mathrm{Tm}$ \\
\hline$\beta$ & 0.99463369 & 0.98680122 & 0.97031293 & 0.92805089 & \\
\hline$\gamma$ & 9.6656380 & 6.1752688 & 4.1347452 & 2.6848995 & \\
\hline$\eta$ & 0.00314 & -0.0124 & -0.0447 & -0.1249 & \\
\hline$h$ & 12 & 12 & 12 & 12 & \\
\hline$h f$ & 4.4332697 & 4.3983589 & 4.3248675 & 4.13649760 & $\mathrm{MHz}$ \\
\hline$R$ & 128.45798 & 128.45798 & 128.45798 & 128.45798 & $\mathrm{~m}$ \\
\hline
\end{tabular}

\section{Low-Energy Gold Parameters at RHIC Injection}

\begin{tabular}{|c|c|c|c|c|c|}
\hline & Energy 1 & Energy 2 & Energy 3 & Energy 4 & Unit \\
\hline$Q$ & 79 & 79 & 79 & 79 & \\
\hline$m$ & 183.433312 & 183.433312 & 183.433312 & 183.433312 & $\mathrm{GeV} / c^{2}$ \\
\hline$W$ & 8.0688664 & 4.8188664 & 2.9188664 & 1.5688664 & $\mathrm{GeV} / N$ \\
\hline$c p$ & 8.9517032 & 5.6741070 & 3.7357048 & 2.3201272 & $\mathrm{GeV} / N$ \\
\hline$E$ & 9.000 & 5.750 & 3.850 & 2.500 & $\mathrm{GeV} / N$ \\
\hline$B \rho$ & 74.460184 & 47.197169 & 31.073558 & 19.298797 & $\mathrm{Tm}$ \\
\hline$\beta$ & 0.99463369 & 0.98680122 & 0.97031293 & 0.92805089 & \\
\hline$\gamma$ & 9.6656380 & 6.1752688 & 4.1347452 & 2.6848995 & \\
\hline$\eta$ & -0.00880 & -0.0243 & -0.0566 & -0.1368 & \\
\hline$h$ & 360 & 363 & 369 & 387 & \\
\hline$h f$ & 27.9995981 & 28.0106014 & 27.9978265 & 28.0846416 & $\mathrm{MHz}$ \\
\hline$\delta C$ & 0 & 0 & 0 & 0 & $\mathrm{~mm}$ \\
\hline
\end{tabular}




\section{References}

[1] David R. Lide (Editor-in-Chief), Handbook of Chemistry and Physics, 80th Edition, 1999-2000, CRC Press LLC, 1999, pp. 1-10 through $1-12$.

[2] M.A. Zucker and R.A. Dragoset (2000). Elemental Data Index (Version 1.1), [Online]. Available: http://physics.nist.gov/EDI [2000, September 6]. National Institute of Standards and Technology, Gaithersburg, MD.

[3] D.E. Groom, et al., The European Physical Journal C 15, 73 (2000)

[4] K.A. Brown, C. Gardner and P. Thieberger, "Rest Mass of Fully Stripped Ions in RHIC: Updated Values", C-A/AP/Note 293, October 2007.

[5] S.Y. Lee, "Accelerator Physics", World Scientific, 1999, pp. 229-230

[6] E.J. Bleser, "Where are the AGS Magnets", Accelerator Division Technical Note 215, May 20, 1985.

[7] C.J. Gardner, "Notes on Orbit Equations in the AGS", C-A/AP/Note 164, September 2004.

[8] R. Thern, "Booster Dipole Production Measurements", Booster Technical Note 190, March 13, 1991.

[9] W. Fischer and S. Peggs, "RHIC Parameters", Revision of 3/18/97.

[10] D.E. Groom, et al., The European Physical Journal C 15, 685 (2000)

[11] C.J. Gardner, et al, "Setup and Performance of the RHIC Injector Accelerators for the 2007 Run with Gold Ions", Proceedings of PAC07, p. 1862.

[12] P. Thieberger, et al, "Improved Gold Ion Stripping at 0.1 and 10 $\mathrm{GeV} /$ nucleon for the Relativistic Heavy Ion Collider", Phys. Rev. ST Accelerators and Beams 11, 011001 (2008). 